Purpose To evaluate adherence to tamoxifen and to identify the most important DTPs in patients with breast cancer on adjuvant endocrine treatment.

Materials and Methods A prospective study was conducted in a university hospital specialising in women's health. Over 6 months patients with breast cancer were included if they were on adjuvant endocrine treatment for at least 1 month. All were interviewed by the pharmacist (Minnesota model). The instrument used to evaluate adherence was the Morisky-Green test.

Results Forty-one patients were included (mean age 55.0 years; ranging from 34-78). In the first visit, the pharmacist identified 82 DTPs (mean: $2.0 \pm 1.1 \mathrm{DTPs} /$ patient), $63.4 \%$ related to drug safety. The adherence to oral endocrine treatment was $36.6 \%$; according to the Morisky-Green test; among the non-adherent patients $92.3 \%$ were non-intentional (mostly by forgetting to take doses of tamoxifen). The patient's average time on endocrine therapy was $24.9 \pm 17.6$ months.

Conclusions We observed that the DTPs are present in oral endocrine therapy and adherence to this treatment can be considered inappropriate. The results obtained may contribute to the development of strategies in pharmaceutical care to improve adherence to oral endocrine therapy and decrease DTPs in breast cancer patients using tamoxifen.

No conflict of interest.

\section{OHP-004 ADHERENCE TO ANTIRETROVIRAL TREATMENT}

doi:10.1136/ejhpharm-2013-000276.378

F Gutiérrez Nicolás, MM Viña Romero, G Callejon Callejon, P Diaz Ruiz, FJ Merino Alonso. Hospital Ntra. Sra. de Candelaria, Pharmacy, Santa Cruz de Tenerife, Spain

Background Knowledge of the patient's adherence to antiretroviral treatment is extremely useful for monitoring HIV infection. However to measure this reliably is not easy. Several methods have been proposed to calculate adherence, each with its advantages and disadvantages.

Purpose To compare three of the available methods for assessing medicines adherence. To determine the factors associated with nonadherence to highly active antiretroviral treatment (HAART) in HIV/AIDS patients.

Materials and Methods Non-interventional and longitudinal study of patients diagnosed with HIV/AIDS who received HAART (May-June 2010). Three methods for evaluating medicines adherence were studied prospectively: Recording medicines dispensed (RD) from the Pharmacy Department; SMAQ (simplified medicines adherence questionnaire) interview; SMAE (scale for medicines adherence evaluation) interview. We recorded: demographic data (age, sex); years in treatment and daily doses of medicines.

Results $85.2 \%$ (104) of patients were males and mean age was 46 years (S8.9) with an average treatment time of 8.7 years (S4.6). $79 \%$ of patients have had a change in their medicines at some point in the treatment.

The percentage of patients with greater than $95 \%$ adherence was: $77.0 \%(\mathrm{RD}), 62.3 \%$ (SMAQ) and $79.4 \%$ (SMAE).

By all measures of adherence patients with a single dose of medicine daily (SDM) were more adherent than twice-daily medicines (TDM): RD: $84 \%$ vs. $70 \%$ ( $p=0.0781$ ); SMAQ: $70.1 \%$ vs. $49.2 \%$ $(p=0.0189) ;$ ESPA: $85.9 \%$ vs. $69.2 \%(p=0.0283)$ respectively. Patients who had been on HAART between 6-10 years had an adherence of $77.1 \%$, while it was $65.8 \%$ for those treated $1-5$ years and for patients with over 10 years of treatment, it was $40.8 \%$ $(p=0.002)$. Similar results with other measures.

Conclusions Since there is currently no ideal method to determine adherence to treatment, it is important to combine several methods depending on patient characteristics to obtain a measure as real as possible. Years with HAART reduces adherence and SDM regimens schedules appear to have better adherence than TDM regimens. This may affect treatment efficacy positively in the long term.

No conflict of interest.

\section{OHP-005 ADVANTAGES AND DISADVANTAGES OF AN ELECTRONIC PRESCRIBING SYSTEM. ASPECTS TO CONSIDER DURING PHARMACIST VALIDATION.}

doi:10.1136/ejhpharm-2013-000276.379

B Corcóstegui Santiago, MJ Martinez Bengoechea, 0 lbarra Barrueta, A Santos Ibañez, E Ardanza Aramburu, E Ibarra Garcia, I Palacios Zabalza, I Ibarrondo Larramendi, M Bustos Martinez. Hospital Galdakao-Usansolo, Hospital Pharmacy, Galdakao, Spain

Background An electronic prescribing system (EPS) improves the prescription-validation-administration sequence and reduces errors. Nevertheless new questions can appear and it is interesting to take them into account.

Purpose To describe positive and negative aspects that the implementation of an EPS produces in a physician when he/she prescribes, in a nurse during the administration of the drugs and in the pharmacist when he/she validates.

Materials and Methods We recorded the advantages and disadvantages identified by pharmacists as seen by different professionals from the introduction in January 2010 of an EPS.

\section{Results}

Positive aspects for the pharmacist: real-time validation (it avoids administration errors and facilitates communication between healthcare professionals); no unreadable or incomplete prescriptions, chance to cheque nurse records (administration time, observations and incidents); quick access to ambulatory care and other hospital admissions medicines records; ability to see and change drug administration rates and information about the drugs is instantly available from databases. Physician: availability of protocols; rapid access to the hospital formulary, automatic drug changes, automatic allergy alerts. Nurse: drugs appear automatically on the administration records, they can request medicines directly from the prescription screen

Negative aspects for the pharmacist: repeated validation is required of unchanged prescriptions; errors can be made if the medicine is changed (e.g. duration of treatment). Physician: errors due to lack of knowledge of trade names (e.g. insulin); the existence of protocols can lead to incorrect prescriptions (e.g. for elderly people); errors due to ignorance of the programme (former frequencies of administration are retained); need to delete old prescriptions. Nurse: they cannot change the administration schedule; some services don't use yet the EPS.

Conclusions The implementation of EPS improves many aspects for all the health professionals involved. Pharmacist validation is more complete, real time and faster. It is necessary to know the programme well to detect new errors as they arise in order to correct them.

No conflict of interest.

\section{OHP-006 ADVERSE EFFECTS OF DAY-HOSPITAL CANCER TREATMENT MONITORED AT HOME: CREATION OF A PHYSICIANÑPATIENT LOGBOOK}

doi:10.1136/ejhpharm-2013-000276.380

${ }^{1} \mathrm{~F}$ Slimano, ${ }^{2} \mathrm{C}$ Catoire, ${ }^{1} \mathrm{~L}$ De Mestier, ${ }^{3} \mathrm{~A}$ Thierry, ${ }^{1} \mathrm{~J}$ Volet, ${ }^{1} \mathrm{~L}$ Petit, ${ }^{2} \mathrm{~F}$ Burde, ${ }^{1} \mathrm{O}$ Bouche. 'University Hospital, Medicine Ambulatory Unit - Oncology-Hematology, Reims Cedex, France; 'University Hospital, Pharmacy, Reims Cedex, France; 'University Hospital, Center for Research and Clinical Investigation, Reims Cedex, France

Background Most anticancer drugs bring adverse effects (AEs) occurring during treatment-free intervals (TIs) while the patient is at home. A significant difference exists between AEs that really 